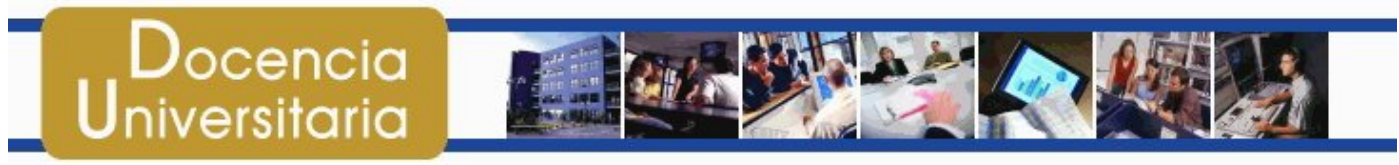

Revista Digital de Investigación en Dacencia Universitaria / Año 3 - N²- Dic. 2007

\title{
YO SOY: Expectativas, imaginación y realidad en la relación profesor - alumno en la universidad
}

\author{
I AM: Expectations, imagination and reality in the teacher-student \\ relationship at the university
}

\section{Fernando Loyola}

\section{Resumen}

El presente artículo aborda la importancia de comprender el rol de la conciencia de uno y la del Otro como elemento fundamental dentro de la dinámica del proceso de enseñanza - aprendizaje, centrándose específicamente en los casos de alumnos adultos que trabajan.

A partir de este marco, se propone un modelo que consta de cinco fases: reconocer, identificar, validar, responder y partir. Estas etapas se encontrarían presentes en toda relación docente alumno situada en el contexto del curso que ambos construyen, sugiriéndose que el éxito o fracaso de la misma podría impactar directamente en los resultados del proceso de aprendizaje.

Palabras clave: vínculo profesor - alumno; educación para adultos; autoconciencia docente.

\begin{abstract}
This article covers the importance of understanding the conscience of oneself and the conscience of the other as a fundamental element within the dynamics of interaction of the teaching-learning process, specifically focusing on the case of adult students who work.

Starting from this framework, the article proposes a five phase model: to recognize, to identify, to validate, to respond and to part. These phases will be present in every teacher-student relationship in the context of the course that they build together, suggesting that the success or the failure during its transition could directly impact the results of the learning process.
\end{abstract}

Key Words: bond between teacher - student; education for adults; teacher self-conscience. 


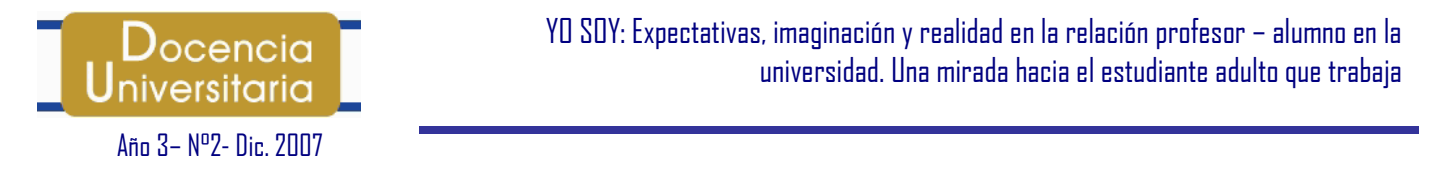

\section{El animal frente al espejo}

Son muy pocos los animales que pueden reconocerse al colocarse un espejo delante de ellos. Al contrario de lo que muchos de nosotros seríamos capaces de hacer -es decir, reconocernos a nosotros mismos y empezar a buscar imperfecciones o perfecciones, según el ángulo desde el que deseáramos vernos, la mayoría de los animales reconocería a otro delante de ellos; un congénere que podría despertar en ellos su curiosidad, su interés, su temor, e incluso su agresividad ${ }^{1}$

Tomémonos la libertad de utilizar este tópico científico para construir una pregunta y, luego, una metáfora. En primer lugar, al contar los animales con la capacidad de reconocerse en una imagen externa, ¿podríamos tener la capacidad de confundir esta imagen, y vernos en quienes no somos? Es decir, si los animales que no se reconocen a sí mismos ven en el espejo a otro animal, ¿podríamos nosotros ver un espejo en el que realmente hubiera otra persona? Por supuesto, no estamos empleando un sentido literal, sino hallándonos en el campo de la comparación utilitaria: ¿Somos capaces de comprender que tenemos a un Otro delante nuestro, alguien que es potencialmente distinto a nosotros y, por lo tanto, todo un reto para nuestros recursos? ${ }^{2}$.

\section{Ese Otro que no soy Yo}

Recuerdo con claridad a aquel profesor que, al borde de la desesperación, alzaba la voz para que todo el salón pudiese escucharlo decir “¿Cómo es posible que no entiendas? ¡Si está clarísimo!” a uno de mis compañeros, que desde esa tarde no volvió a realizar preguntas en clase, asintiendo tímidamente cada vez que el mismo profesor le solicitaba confirmar que hubiera comprendido totalmente la lección.

Quizás uno de los mayores retos de la práctica docente sea el auténtico reconocimiento de ese Otro que tenemos enfrente. Ese Otro que no soy Yo, y que tampoco debería serlo $^{3}$. Un Otro con experiencias, opiniones, intereses y recursos que nos resultan completamente desconocidos en el primer día de clases, y que para algunos podrían seguirlo siendo al final de un ciclo o de un año.

\footnotetext{
${ }^{1}$ Sanpedro, 2006; Bard, Todd, Bernier, Love y Leavens, 2006; Bradshaw, 1998; Dawkins, 2001; Griffin y Speck; 2004.

2 Derrick, Carr y Ponton, 2005; Vella, 1994)

${ }^{3}$ Muñoz - Yunta y Palau - Baduell, 2004.
} 


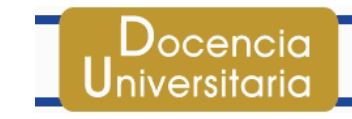

A lo largo de nuestro trabajo con el equipo de docentes de la División de Estudios Profesionales para Ejecutivos (EPE) ${ }^{4}$ de la Universidad Peruana de Ciencias Aplicadas (UPC), hemos abordado el tema desde diferentes aristas, reconociendo más de una vez su valor capital en la definición del estilo docente que requiere el adulto que, en el marco de su formación profesional de pregrado, trabaja. Esta población posee una serie de características que la distinguen del alumno adolescente o adulto joven y, por lo tanto, merece una revisión de los procesos educativos involucrados en el contexto de su aprendizaje $e^{5}$.

Así, por ejemplo, en un estudio realizado con los profesores mejor calificados por sus alumnos, en el que buscábamos hallar los elementos que convertían a algunos docentes en "personajes positivamente memorables", reconocimos de inmediato la importancia que estos le brindaban a la personalización de sus estudiantes y al reconocimiento de las diferencias individuales como factores que potenciaban el desarrollo de sus sesiones de clase y de sus cursos en sí ${ }^{6}$.

\section{Ese Otro que soy Yo}

Si reconocer la naturaleza del Otro supone dificultades, reconocer nuestra propia naturaleza de "Otro" ante los ojos de nuestros alumnos podría resultar un ejercicio aún más interesante y complejo: ser capaz de proyectarse hacia los ojos del alumno (en lugar de mirarse con los propios), de tal manera que uno pueda ser conciente de las posibles interpretaciones de nuestras palabras y nuestros actos ${ }^{7}$.

Al trabajar con el equipo de profesores a tiempo completo de EPE, en reuniones semanales en las que tocamos temas vinculados a la excelencia académica, hemos concluido que un factor que frecuentemente interfiere en la relación docente - alumno es la mala interpretación de los mensajes que el primero envía al segundo ${ }^{8}$. Volvamos a la metáfora del espejo: ¿No será que esperamos que ese Otro que tenemos delante nuestro nos mire con complejidad mientras reconoce a la perfección la intención oculta detrás de cada uno de nuestros movimientos? Sería posible si estuviésemos frente a

\footnotetext{
${ }^{4}$ La División de Estudios Profesionales para Ejecutivos (EPE) es una unidad académica y administrativa de la corporación UPC que ha sido constituida con el fin de dar soporte a las carreras universitarias para personas que trabajan. EPE define su misión de la siguiente manera: "Estamos comprometidos en la trasformación de adultos con experiencia laboral en profesionales y líderes íntegros e innovadores, capitalizando toda su experiencia, talento y potencial de desarrollo” Página Web: http://www.upc.edu.pe

${ }^{5}$ Knowles, 1984; Long, 1990; Vella, 1994.

${ }^{6}$ Buitrón y Loyola, 2007a.

${ }^{7}$ Loyola, 2007a.

${ }^{8}$ Buitrón y Loyola, 2007b.
} 


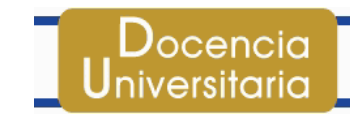

nuestra propia imagen, pero ¿qué podríamos decir de un grupo de personas distintas a uno?

Comprender nuestra naturaleza de "Otros" nos permitirá abrir las puertas hacia la comprensión verdadera.

\section{Entre el ser y el no ser: La relación}

Una vez dimensionado el tema, y siendo capaces de aceptar que somos parte, día a día, de una serie de interacciones que tienen correspondencia en situaciones y respuestas de espectro casi infinito, estamos listos para empezar a construir una relación con esos otros, con quienes compartimos el espacio físico en el que se desarrollan nuestras clases: una relación en la que expectativas, experiencias previas, sutilezas y discrepancias moldearán la forma en la que el curso discurrirá y, finalmente, llegará o no a dar los resultados esperados.

Para comprender con mayor profundidad a qué nos estamos refiriendo, podríamos realizar una división -arbitraria y artificial- del vínculo profesor - alumno en cinco grandes momentos, en los cuales, de generar las condiciones necesarias, deberíamos construir un "nosotros".

Así, deberíamos estar dispuestos, en toda actividad docente, a reconocer, identificar, validar, responder y partir; cinco verbos que constituyen cinco acciones específicas que podrían aportar una diferencia significativa en nuestro desempeño.

\section{Reconocer}

El primer paso es la realización del proceso que hemos venido comentando: Reconocernos y reconocerlos. Es decir, comprender la esencia de los actores de la interacción y las posibilidades que estos aportan a la relación.

Las siguientes serían algunas de las preguntas que podríamos hacernos:

\begin{tabular}{|l|l|}
\hline \multicolumn{1}{|c|}{ Él / Ella } & \multicolumn{1}{c|}{ Yo } \\
\hline - ¿Quién es? & $\bullet$ ¿Quién soy? \\
- ¿Por qué está aquí? & $\bullet$ ¿Por qué estoy aquí? \\
- ¿Qué espera de mí? & ¿ ¿Qué espero de él? \\
- ¿Qué me dice? & ¿QQué le digo? \\
\hline
\end{tabular}

Las respuestas que demos a estas y otras preguntas similares resultarán cruciales para construir la relación, ya que, trabajadas correctamente, nos ayudarán a comprender las 


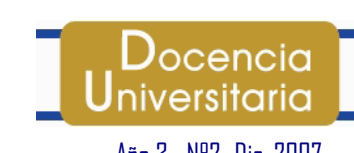

expectativas (propias y ajenas) que habitan en nuestros salones de clase. Esto nos permitirá modularlas y enfocarlas.

\section{Identificar}

Una vez realizada la "personificación” de los actores de esta relación, deben identificarse las actitudes y aptitudes que se interrelacionan en clase. Preguntas como las siguientes podrían ayudarnos en esta tarea:

\begin{tabular}{|c|c|}
\hline Él / Ella & Yo \\
\hline 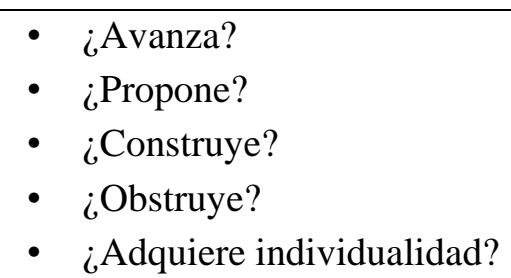 & $\begin{array}{l}- \text { ¿Avanzo? } \\
-\quad \text { ¿Propongo? } \\
\cdot \text { ¿Construyo? } \\
-\quad \text { ¿Obstruyo? } \\
\cdot \quad \text { ¿Adquiero individualidad? }\end{array}$ \\
\hline
\end{tabular}

De este modo, las imágenes que empezamos a reconocer como uno o como otros en el espejo del proceso de enseñanza, se van definiendo con mayor nitidez y precisión. Esta es una tarea que no puede detenerse; se trata, a diferencia del análisis de una escena estática, de la observación de procesos sobre la marcha. Por lo tanto, el fin último de esta tarea sería la intervención focalizada y la construcción continua del clima propicio para el desarrollo de nuestros cursos.

\section{Validar}

Una de las razones por las que utilizamos un espejo es la validación de alguna de nuestras creencias o suposiciones. Por ejemplo, nos miramos para saber si estamos bien afeitados, si lucimos enfermos, o si nos quedan bien los pantalones nuevos que nos hemos comprado. Lo mismo ocurre con nuestros alumnos. Ellos esperan ver en nosotros el reflejo de su desempeño. Esta condición nos acerca, en la fantasía de más de uno, al espejo mágico del cuento, que dice solamente la verdad y que siempre está dispuesto a responder. La pregunta que surge es inminente: ¿Estamos dispuestos a asumir este rol? Para aclararlo, es necesario plantear algunas preguntas que podrían ser de utilidad: 


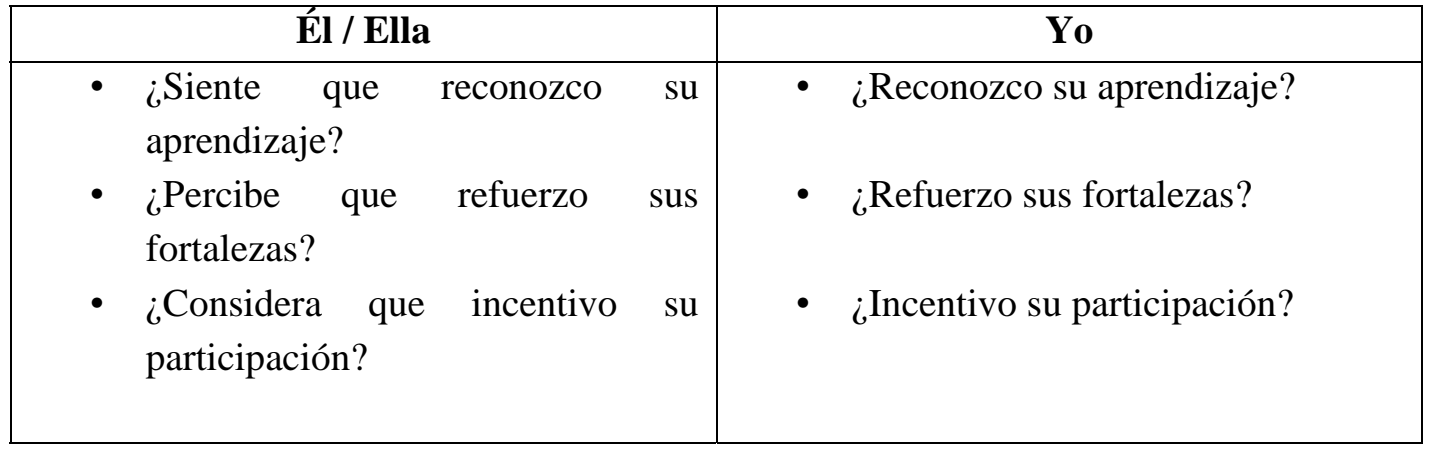

En tanto nuestras respuestas sean más favorables a cada una de las preguntas, muy probablemente estaremos cumpliendo ese rol de espejo fiel y objetivo.

\section{Responder}

Al poner sobre la mesa el tema de las preguntas durante (y después de) la clase, un profesor que no había sido evaluado positivamente por sus alumnos respondió: "Sí, son algo inevitable y hay que saber sortearlas". Por su parte, y ante el mismo tema, otro colega, con calificaciones más altas, comentó que "es lo que hace la diferencia entre una clase fluida y una difícil: más preguntas son más oportunidades”. ¿Es posible que la actitud que tengamos ante las preguntas de nuestros alumnos sea un factor decisivo para definir o desdibujar el clima de nuestras sesiones? Aparentemente sí.

Esta vez, el ejercicio nos propone preguntarnos sobre las preguntas, por lo que podríamos reflexionar en torno a temas como los siguientes:

\begin{tabular}{|c|c|}
\hline Él / Ella & Yo \\
\hline $\begin{array}{ll}\text { - } & \text { ¿Me pregunta o simplemente } \\
& \text { pregunta? } \\
\text { - } & \text { ¿Su curiosidad se ve satisfecha? } \\
\text { - } & \text { ¿Espera respuestas? } \\
\text { - ¿Recibe las respuestas incluso } \\
\text { antes que las preguntas? }\end{array}$ & $\begin{array}{ll}- & \text { ¿Le respondo o simplemente } \\
& \text { respondo? } \\
\text { - } & \text { ¿Satisfago su curiosidad? } \\
\text { - } & \text { ¿Lo impulso a esperar respuestas? } \\
\text { - } & \text { ¿Genero las respuestas incluso } \\
& \text { antes que las preguntas? }\end{array}$ \\
\hline
\end{tabular}

Y, nuevamente, nos enfrentamos a la visión metafórica del alumno mirándose en nosotros mientras dice “espejito, espejito”. ¿Qué respuestas damos ante esta situación particular? 


\section{Partir}

Considerar que la relación profesor - alumno tiene límites claros, que son los mismos dados por el cronograma del curso, podría resultar una visión limitada de las cosas. Sobre todo si consideramos las redes emocionales que se tejen entre sus dos actores, los contenidos del curso, la carrera, e, incluso, la institución.

Revisemos las siguientes preguntas y miremos nuestras experiencias. De esta forma, podremos reconocer si, al final de cada experiencia de enseñanza, cerramos con reflexiones como:

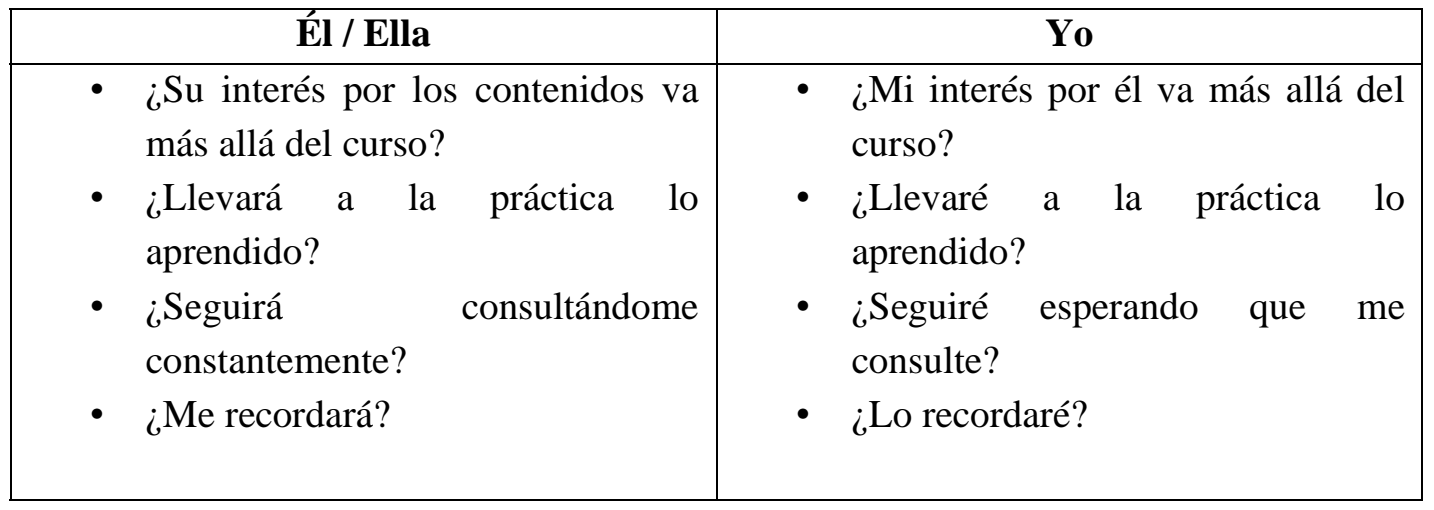

El balance es importante si nos permite cerrar adecuadamente nuestra relación con el grupo humano con el que hemos trabajado durante semanas o meses. Incluso, nos ayuda a definir la forma en que la interacción podría continuar en un fuero distinto.

\section{El fin, que es el principio}

Lo interesante empieza al preguntarse qué es lo que viene después. La respuesta es muy sencilla, tanto como la cuestión que nos condujo a la elaboración de este texto: Una vez concluida esta secuencia, se inicia un nuevo ciclo, compuesto por esas mismas cinco fases continuamente enriquecidas, que trazan un espiral de experiencia que nos va convirtiendo en mejores docentes; esto, naturalmente, siempre y cuando estemos dispuestos a mirarnos en esta dinámica de espejos y reflejos que supone la conducción de un proceso de aprendizaje. 


\section{Bibliografía}

BARD, Kim; TODD, Brenda; BERNIER, Chris; LOVE, Jennifer; y LEAVENS, David

2006 Self-Awareness in Human and Chimpanzee Infants: What is Measured and What is Meant by the Mark and Mirror Test? Infancy 9 (2). pp.191-219.

BRADSHAW, R. H.

1998. Consciousness in Nonhuman Animals: Adopting the Precautionary Principle. Journal of Consciousness Studies 5 (1). pp. 08-14.

BROWNE, Derek

2004 Do Dolphins Know Their Own Minds? Biology and Philosophy 19 (4). pp.633653.

BUITRÓN, Sigrid y LOYOLA, Fernando.

2007a Perfil del docente exitoso de EPE: Una aproximación a través de la experiencia. Documento de trabajo del Área de Calidad Educativa EPE - Universidad Peruana de Ciencias Aplicadas, Mayo de 2007.

2007b Equipo de Mejora de la Calidad Docente EPE: Actas de Reunión. Octubre Noviembre 2007. Documento de trabajo del Área de Calidad Educativa EPE Universidad Peruana de Ciencias Aplicadas

DAWKINS, Marian

2001 Who Needs Consciousness? Animal Welfare Supplement. 10. pp.19- 29.

DERRICK, Gail; PONTON, Michael y CARR, Paul

2005 The Relationship between Resourcefulness and Persistence in Adult Autonomous Learning. En: Adult Education Quarterly, Vol. 55, No. 2, 116-128 (2005)

DRI, Rubén

2001 Dialéctica de la conciencia a la autoconciencia, en Razón y Revolución, Nro. 8. Primavera de 2001. Reedición electrónica.

GRIFFIN, Donald y SPECK, Gayle.

2004 New Evidence of Animal Consciousness.

Animal Cognition 7 (1). pp. 5-18. 
Аก̃̃ 3- N02- Dic. 2007

KNOWLES, Malcolm

1984 The Adult Learner: A Neglected Species. 3ra.ed. Houston: Gulf Publishing Co.

LONG, Douglas

1990 Learner Managed learning. The Key to Life Long Learning and Development. New York: Kogan Page

LOYOLA, Fernando

2007a Reflexiones en torno a la relación con el alumno adulto. Documento de trabajo del Área de Calidad Educativa EPE.

MUÑOZ-YUNTA, J.A. y PALAU-BADUELL, M.

2004 Ontogenia de la conciencia: Cómo se construye el cerebro cognitivo. En: Revista de Neurología, 2004, 38, Supl 1, s3 - s8

SAMPEDRO, Javier

2006 Una elefanta ante el espejo. Madrid. En: Diario El País, 31/10/2006

VÁSQUEZ, Eduardo

2002 Tesis fundamental de "el mundo invertido". En: Dikaiosyne, No. 9 Revista de filosofía práctica. Universidad de Los Andes Mérida, Venezuela, Diciembre de 2002.

VELLA, Jane

1994 Learning to Listen, Learning to Teach: The Power of Dialogue in Educating Adults. San Francisco: Jossey - Bass.

\section{Para citar este documento, puede utilizar la siguiente referencia:}

LOYOLA, Fernando (2007). “ YO SOY: Expectativas, imaginación y realidad en la relación profesor - alumno en la universidad. Una mirada hacia el estudiante adulto que trabaja” [artículo en línea]. Revista Digital de Investigación en Docencia Universitaria (RIDU) Año 3 - $N^{\circ} 2$-Diciembre 2007. [Fecha de consulta: dd/mm/aa]. <http://beta.upc.edu.pe/calidadeducativa/ridu/2007/ridu4 3FL.pdf $>$ 
Añ̃ 3- N²- Dic. 2007

\section{Fernando Loyola Ángeles}

floyola@upc.edu.pe

Licenciado en Psicología Social (PUCP) con especialidad en Psicología Organizacional. Máster en Gestión Estratégica del Factor Humano (EOI - España). Diploma en Gestión Estratégica del Factor Humano con especialización en Consultoría Organizacional (Escuela de Postgrado - UPC).

Docente de la Facultad de Estudios de la Empresa de la UPC en los cursos de Gerencia Avanzada. Además, ha ejercido la cátedra en temas de comportamiento organizacional, ética para los negocios y técnicas de innovación social.

Actualmente se encuentra a cargo de la Dirección Adjunta de Calidad Educativa de la UPC. 